\title{
Temporal summation in human vision: Simple reaction time measurements
}

\author{
TAKEHIRO UENO \\ Osaka City University, Sumiyoshi-ku, Osaka 558, Japan
}

\begin{abstract}
Simple reaction time (RT) was measured as a function of stimulus intensity for a brief light pulse $(1 \mathrm{msec})$ and a long one $(300 \mathrm{msec})$. Target size, retinal position, and adapting luminance of the stimulus were varied parametrically, and the luminance value required to produce a $R T$ of 50 msec greater than the asymptotic RT was calculated to obtain the critical duration or limit of time-intensity reciprocity. It was found that: the critical duration, even at the fovea, tends to increase with decreasing target size; the critical duration is shortest at the fovea and increases sharply with distance from the fovea; and as the adapting luminance increases, the critical duration decreases. These findings indicate that the RT technique is a sensitive measure for the stimulus conditions explored.
\end{abstract}

Studies of temporal summation in vision have demonstrated Bloch's law, which states that the luminance $(\mathrm{L})$ and duration $(\mathrm{t})$ of a light stimulus pulse has a reciprocal relationship $(L \times t=C)$ for a constant visual effect. The longest duration at which reciprocity is found has been called the critical duration (Hartline, 1934). Psychophysical studies using threshold measures have shown that critical duration is about 100 msec for foveal (Herrick, 1956; Roufs, 1972) as well as peripheral vision (Barlow, 1958; Baumgardt \& Hillmann, 1961). In contrast to this, recent reaction time (RT) experiments have frequently demonstrated the order of $10 \mathrm{msec}$ (Grossberg, 1970; Kietzman \& Gillam, 1972; Mansfield, 1973; Ueno, 1976b). This difference in critical duration between these two types of experiments may result from different levels in stimulus intensity.

It appears that the relation between $L$ and the reducible part of $\mathrm{RT}$ is primarily determined by distal retinal processes associated with the photoreceptors. The present experiments were designed to study temporal summation at suprathreshold levels by using RT as a measure of sensory integration. Summation for suprathreshold stimuli is an interesting adjunct to that at threshold, because an analysis of the intensity-response functions in vision provides the evidence that the visual system behaves nonlinearly at intensity levels substantially above threshold, suggesting slightly different integrative processings from threshold level (Lipetz, 1969). The

Part of this study was supported by a Grant in Aid for Scientific Researches (General D, No. 161042, 1976), Ministry of Education. The author wishes to thank Mr. M. Ito for his design of the random generator. Requests for reprints should be sent to Takehiro Ueno, Department of Psychology, Faculty of Letters, Osaka City University, Sumiyoshi-ku, Osaka 558, Japan. major advantage of the RT method, over the use of threshold previously employed, is that it permits the examination of temporal summation for suprathreshold stimulus pulses without the need to resort to brightness scaling or matching.

Although there are many variables that do affect the critical duration, three variables, i.e., target size, retinal locus, and adapting luminance level, were investigated in the present study. It was found that the RT measure works, because RT was very sensitive to stimulus energy for the conditions explored.

\section{GENERAL METHOD}

\section{Apparatus}

The main optical system has been described previously (Ueno, 1976a, 1976b). The light source was a glow-modulator tube (Sylvania, R1131C), operated at $40 \mathrm{~mA}$ and located at a distance of $50 \mathrm{~cm}$ in front of a box. The box was $33 \mathrm{~cm}$ in length, $30 \mathrm{~cm}$ in width, and $44 \mathrm{~cm}$ in height, with a window $(8 \mathrm{~cm}$ in length and $7 \mathrm{~cm}$ in width) cut from the wall opposite to that where the light stimulus was presented. Therefore, the subject could directly view light flashes through the window. Light from the glowmodulator tube, after passing a diaphragm stop, filter holders, and a lens system, was focused onto a small ground-glass plate fastened to the box wall in such a way that it produced a sharp circular image. The transilluminated light stimulus was directly in front of the subject and at the level of his eyes, and its luminance level was controlled by a series of calibrated Kodak Wratten neutral density filters (No. 96).

The glow-modulator tube was electronically gated. The waveform and duration of the light pulse were controlled by a sixchannel time regulator (Sanwa, DTR-6) driven by a $100-\mathrm{kHz}$ crystal clock. The waveform was nearly square when displayed on an oscilloscope, and its rise and decay times were less than $30 \mu \mathrm{sec}$. A random generator was employed to control the variable foreperiod between the warning signal and stimulus onset.

Reaction time from stimulus pulse onset to the button-pushing response was measured by a TKK digital counter (TW-7010A) and recorded by a TKK digital printer (DP-18). 


\section{General Procedure}

The subject was seated in front of the light-tight box and was positioned at a bite-board. Then he was instructed to respond as quickly as possible to each flash by pushing a microswitch with his right fingertip. After a period of about 10 min of dark adaptation. the experiment was begun. The light pulses were delivered with a random (exponential) foreperiod of $1-7 \mathrm{sec}$ (average $3 \mathrm{sec}$ ) between the audio warning signal and the stimulus onset. The intertrial interval between them was 4.2-11.5 sec.

\section{General Design}

The experimental paradigm was very similar to the previous one (Ueno. 1976a, 1977), except that in this study only two durations, specified below. were employed. The main purpose of the present experiment was to obtain a family of the RT vs. L curve at several levels of the above-mentioned variables, taking stimulus duration as a parameter. A block of 18 identical light pulses was presented to find a single representative value of RT to a fixed L level. Data processing for the trial block was as follows: The first RT was skipped, and then the geometric mean of the remaining 15 RTs was computed after excluding the longest and shortest of them.

\section{Estimator of the Critical Duration}

The present experiment required estimates of the critical duration, $t_{c}$, for several levels at each of the three variables. It would be a very time-consuming experiment if we were to obtain $t_{c}$ by using the previous paradigm (Ueno, 1976a, 1977). Therefore, the following economical measure (Krauskopf \& Mollon, 1971; Uetsuki \& Ikeda, 1971) was used:

$$
\log t_{c}=\log L_{s}-\log L_{1}+\log t_{s},
$$

where $\mathrm{t}$, and $\mathrm{L}$, represent the duration and threshold luminance of a stimulus short enough for Bloch's law to be valid, and $\mathrm{L}_{1}$ represents the threshold luminance at a long duration for which the relation $\mathrm{L}=$ constant holds.

Equation 1 has its origin in the threshold studies, but it was available for the present purpose. Two pulse durations, 1 and $300 \mathrm{msec}$, were selected. Therefore, Equation 1 is

$$
\log t_{c}=\log L_{、}-\log L_{1} .
$$

The data obtained indicated that the RT vs. L curve with the 300 -msec duration shifts sufficiently to the left as L decreases, compared with the $1-\mathrm{msec}$ duration curve (e.g., see Parts A-C in Figure 1). This finding may justify the extrapolation involved in Equation 2. The luminances $\left(L_{5}\right.$ and $\left.L_{1}\right)$ required to produce a RT value of $50 \mathrm{msec}$ above the asymptotic $\mathrm{RT}_{0}$ were calculated from the !wo RT vs. L curves to examine temporal summation at one suprathreshold level, and then the value of $t_{c}$ was estimated by substituting these calculated $\mathrm{L}_{5}$ and $\mathrm{L}_{\mathrm{l}}$ values into Equation 2.

\section{EXPERIMENT 1}

The purpose of the experiment was twofold. First, it was to examine the effect of target size on the critical duration at foveal vision. Second, it was to inspect, by the same data, the largest critical area at which complete spatial summation (Ricco's law) is found.

\section{Method}

Stimulus condition. The target stimulus consisted of a circular hole on a thin metal mask that was mounted on the groundglass plate on the side toward the subject. The target was projected through an interference filter (half-power bandwidth of
$10 \mathrm{~nm}$ ) with peak transmittance at $640 \mathrm{~nm}$, and its highest luminance was $42 \mathrm{~cd} / \mathrm{m}^{2}$. Thus, the subject could view a red circular target with dark surround through the observational window.

Eight targets were used: $2.7^{\prime}, 4.5^{\prime}, 7.1,13.4^{\prime}, 17.9^{\prime}, 26.8^{\prime}$, $35.7^{\prime}$, and $60^{\circ}$ in visual angle. Two small dim red spots $\left(0.15^{\circ}\right)$ served as fixation points, located svmmetrically on both vertical sides $2^{\circ}$ from the center of the target. The subject was instructed to gaze with his right eye at the midpoint between the fixation points, so that the monocular field was foveally fixated.

Procedure. The subjects served in four sessions, and only one session was run per day. Within one session, two of the eight target stimuli were randomly selected. and two RT vs. L curves were obtained for each targe: under the 1 - and 300 -msec-duration conditions. Consequently. a totai of four curves was collected during one session. Before starting each experimental session, the subject-performed $20 \mathrm{warm}$-up RT responses to the flashes with moderate stimulus entergy at each duration.

For each target, five to eight luminance levels were chosen by a criterion of $100 \%$ delection under each duration condition. The measurement of $\mathrm{R} T \mathrm{~s}$ to these luminances was as follows: First, the experimental order was determined as the sequence $\mathrm{ABAB}$ or $\mathrm{BABA}$ ( $\mathrm{A}$ and $\mathrm{B}$ refer to the 1 - and 300 -msec-duration conditions, respectively); the $\mathrm{L}$ levels chosen were divided randomly into two groups in nearly equal numbers; then one group was assigned to the first $\mathrm{A}$ (or $\mathrm{B}$ ), and the other to the second $\mathrm{A}$ (or $\mathrm{B}$ ); during one $\mathrm{A}$ (or $\mathrm{B}$ ), an irregular but descending sequence of $\mathrm{L}$ levels was used, and for each $\mathrm{L}$ level there was one block of $18 \mathrm{RT}$ trials. If the subjects missed light flashes or made anticipatory responses, the sequence was extended until 18 RTs were obtained. No feedback was given to the subjects concerning the anticipations. A rest period of about $30 \mathrm{sec}$ was taken before new trial block of pulse presentations. During each session, the experimental order of the two targets and the preceding sequences for the 1 - and 300msec durations were randomized for each subject

Subjects. Two well-practiced subjects, A.H. and T.U., served in the experiment. Both subjects had normal vision, except for refractive errors that were adequately corrected by glasses.

\section{Results}

RT vs. L curve. The left-hand parts of Figure 1 (A-C) present an example of the data obtained for subject A.H: the geometric means of RTs are plotted as a function of $\log \mathrm{L}$, taking three target sizes and two durations as a parameter. The curves connecting the data points are the function

$$
\mathrm{RT}=\frac{\mathrm{k}}{\mathrm{L}^{\mathrm{n}}}+\mathrm{RT}_{0},
$$

where $\mathrm{k}$ and $\mathrm{n}$ are constants and $\mathrm{RT}_{0}$ represents the irreducible minimum latency. The reducible latency $\mathrm{RT}-\mathrm{RT}_{0}$ in Equation 3 refers to the intensitydependent component, whereas $\mathrm{RT}_{0}$ refers to the fixed motor response component (Mansfield, 1973; Piéron, 1952; Vaughan, Costa, \& Gilden, 1966).

Equation 3 was fitted to all of the data obtained for subjects A.H. and T.U. The values of $R_{0}$ were graphically estimated from the asymptotes of the RT vs. $\log \mathrm{L}$ curves, such as shown in Parts A-C. By the use of least square method, the values of $k$ and $n$ were then calculated after plotting $\log \left(\mathrm{RT}-\mathrm{RT}_{0}\right)$ against $\log \mathrm{L}$. Parts $\mathrm{D}$ and $\mathrm{E}$ show the estimated values of $n$ and $R T_{0}$ for subject A.H. as a function 

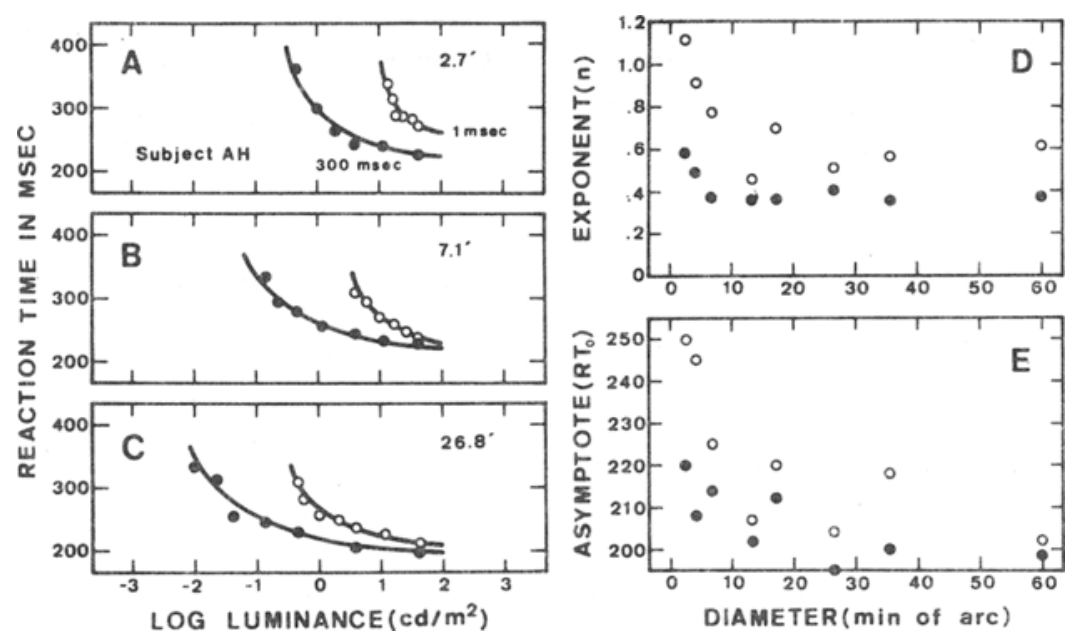

Figure 1. (A-C) Reaction time as a function of log luminance, with target size and pulse duration as a parameter for subject $A$.H. The solid curves drawn through the data points represent the RT vs. $\log L$ curve, Equation 3 , fitted by the method of least squares. Open circles are for 1-msec duration and filled circles for 300-msec duration. (D) Estimated exponent (n) in Equation 3 as a function of target size for subject A.H. Open circles for 1-msec duration and filled circles for 300-msec duration. (E) Estimated asymptote $\left(\mathbf{R T}_{0}\right)$ as a function of target size for subject $\mathbf{A}$.H. The legends in the figure are similar to those in part $D$.

of target size with stimulus duration as a parameter. As expected from the shapes of RT vs. L curve, the $\mathrm{n}$ and $\mathrm{RT}_{0}$ values change systematically with target size: both $\mathrm{n}$ and $\mathrm{RT}_{\mathrm{o}}$ decrease exponentially with the increased size in target; and they are always larger at the 1-msec duration than at the 300 -msec duration. The results for subject T.U. were roughly the same as those of subject A.H.

Critical area and duration. The luminance required to produce a $\mathrm{RT}-\mathrm{RT}_{0}$ of $50 \mathrm{msec}$ was calculated from an individual RT vs. L curve fitted for each subject, and, consequently, two luminance values, one for each subject, were obtained for each stimulus duration and each target size.

Part $\mathbf{A}$ of Figure 2 shows that the means of these luminances are plotted against log area of the target, taking two durations as a parameter. The straight lines running among the data points are Ricco's law, $\mathrm{L} \times \mathrm{A}=\mathrm{C}$, and the relation $\mathrm{L}=\mathrm{D}$, where $\mathrm{A}$ represents stimulus area and $C$ and $D$ are constants. It will be found that the upper limit of complete spatial summation is approximately $10^{\prime}$ at the two durations. Figure $2 \mathrm{~B}$ is a plot of $\log$ critical duration vs. $\log$ area of the target; the critical durations, based on Equation 2, are computed from the difference between the 1-msec duration data (open circles, Figure $2 \mathrm{~A}$ ) and the $300-\mathrm{msec}$ duration data (filled circles, Figure 2A). Of interest is the finding that even at the fovea the critical duration is slightly longer for small target size.

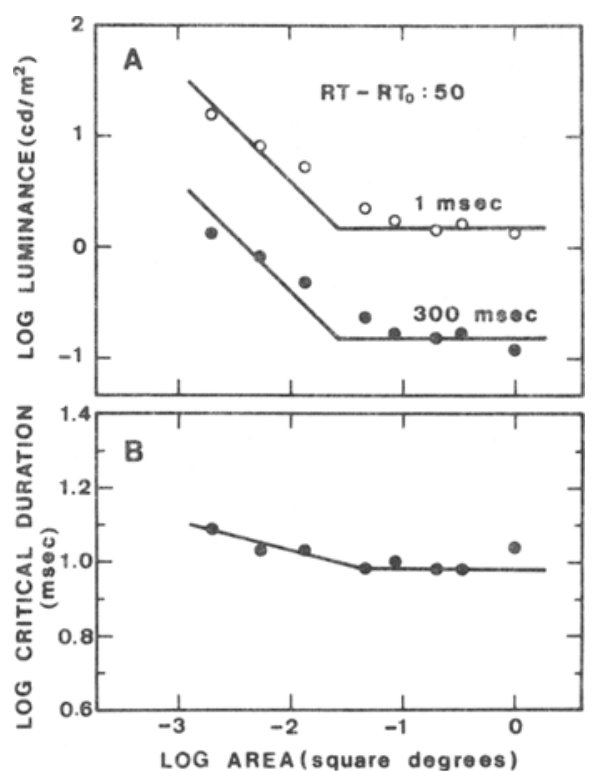

Figure 2. (A) Effect of target size on RT. Luminance required to produce a criterion $R T$ of 50 msec above the asymptotic $\mathbf{R T}_{0}$ level is plotted as a function of target area for short duration (open circles) and long duration (filled circles). Each point represents the average of two subjects, A.H. and T.U. Data for small area are fitted with a line of -1 slope: data for large area are fitted with a horizontal line. (B) Critical duration as a function of target area. The solid line running among the data points is drawn by visual inspection. 


\section{EXPERIMENT 2}

The present experiment was designed to examine the effect of retinal locus on the critical duration for the light pulses that stimulate red cones.

\section{Method}

Stimulus condition. The target stimulus consisted of a $1^{\circ} \mathrm{cir}$ cular image and was projected through a $640-\mathrm{nm}$ interference filter. The highest luminance of the light was $41 \mathrm{~cd} / \mathrm{m}^{2}$.

Ten retinal positions were tested: one was the center of the fovea, and along the horizontal meridian the other nine were at $2.5^{\circ}, 5^{\circ}, 10^{\circ}$, and $19^{\circ}$ in the nasal retina and $2.5^{\circ}, 5^{\circ}, 10^{\circ}$, $15^{\circ}$, and $19^{\circ}$ in the temporal retina. The device for fixating so that the image of the light pulse fell at each of these peripheral positions consisted of a spot of luminous diode, which appeared as a deep red circular image. The spot subtended $0.45^{\circ}$ in visual angle. The subject was instructed to fixate the spot with his right eye. The foveal fixation device was identical with that in Experiment 1.

Plastic dental impressions were made for each subject to obtain a stable position for his head. The subject positioned his head by aligning his teeth with the impressions in the dental place.

Subjects. Two subjects, A.H. and T.U., in Experiment 1 served in this experiment. The experimental order for subject A.H. began from the retinal positions in the temporal retina, including the fovea, whereas for subject T.U., it began from those in the nasal retina.

Procedure. The subjects served in a total of five sessions, and one session was run per day. Within one experimental session, two retinal positions were randomly chosen from the hemiretina prescribed for each subject; then two RT vs. L curves were obtained for each retinal locus, one with the $1-\mathrm{msec}$ duration and the other with the 300 -msec duration. Prior to each session, the subjects were given 20 warm-up trials.

For each retinal position, five to seven luminance levels were determined at each stimulus duration by a criterion of $100 \%$ detection. The measurement procedure of RTs to these luminances was the same as in Experiment 1. After one retinal locus, another was changed by selecting the proper fixation spot. During this interval, the subjects rested for about $2 \mathrm{~min}$. Other aspects of the procedure were identical with Experiment 1.

\section{Results}

RT vs. $L$ curve. An example of the data obtained for subject T.U. is shown in Parts A-C of Figure 3. The geometric means of RTs are plotted as a function of $\log \mathrm{L}$, taking three retinal positions and two stimulus durations as a parameter. The curves in the figure represent Equation 3, fitted to the data by the method of least squares. Parts D and $E$ show that the two parameters, $\mathbf{n}$ and $\mathrm{RT}_{0}$, in Equation 3 for subject T.U. are plotted as a function of eccentricity with pulse duration as a parameter. Both $\mathrm{n}$ and $\mathrm{RT}_{0}$ are lowest at the fovea, or $5^{\circ}$ eccentricity, and they increase with the increased distance from the fovea. This effect is more remarkable at the 1-msec duration. Subject A.H.'s results were similar to those of subject T.U.

Critical duration. The obtained RT vs. L curves were analyzed at the same criterion RT level as in Experiment 1. In Part A of Figure 4, the means of the luminances required to produce a latency $50 \mathrm{msec}$ greater than the asymptotic $\mathrm{RT}_{0}$ are plotted as a function of eccentricity with stimulus duration as a parameter. The luminance increases with distance from the fovea: this effect is more pronounced with the 1 -msec duration. Part B presents a plot of $\log$ critical duration vs. eccentricity; the critical durations are computed from the difference between the 1-msec duration data (open circles, Figure 4A) and the 300-msec duration data (filled circles, Fig-
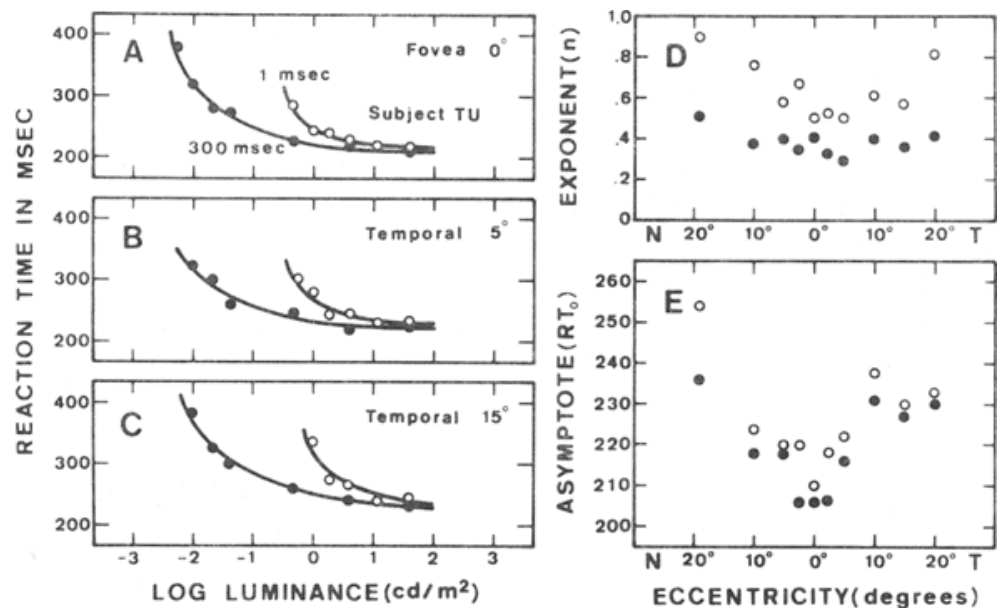

Figure 3. (A-C) Reaction time as a function of log luminance, with retinal position and pulse duration as a parameter for subject T.U. The solid curves drawn through the data points represent the RT vs. log I. curve, Equation 3, fitted by the method of least squares. Open circles are for 1 -msec duration and filled circles for 300-msec duration. (D) Estimated exponent ( $n$ ) in Equation 3 as a function of eccentricity for subject T.U. Open circles are for 1-msec duration and filled circles for 300-msec duration. (E) Estimated asymptote $\left(\mathbf{R T}_{0}\right)$ in Equation 3 as a function of eccentricity for subject T.U. The legends are similar to those in part D. 


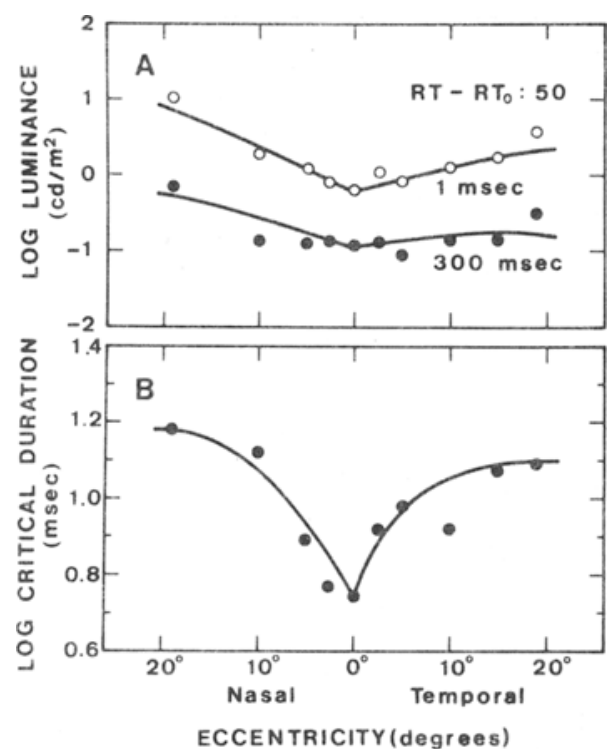

Figure 4. (A) Effect of retinal locus on RT. Luminance required to produce a criterion RT of $50 \mathrm{msec}$ above the asymptotic $\mathbf{R T}_{0}$ level is plotted as a function of eccentricity for short duration (open circles) and long duration (filled circles). Each point represents the average of two subjects, A.H. and T.U. The solid curves drawn through the data points are by visual inspection. (B) Critical duration as a function of eccentricity. The solid curve is drawn by visual inspection.

ure $4 \mathrm{~A}$ ). It will be seen that the critical duration decreases sharply with distance from the fovea.

\section{EXPERIMENT 3}

The purpose of Experiment 3 was twofold. First, it was to investigate the effect of adapting luminance on the critical duration. Second, it was to examine the relation between adapting levels and the specific parameter $L_{0}$ in the hyperbolic $R T$ function developed by Bartlett and MacLeod (1954).

\section{Method}

Stimulus condition. The optical system was modified so as to superimpose a test field upon an adapting field with a silvered half mirror. White light from the glow-modulator tube was used as the test stimulus and focused onto the ground-glass plate mounted to the box wall. The adapting stimulus was a steady-state white light from a Cabin projector lamp, which was imaged by the half mirror on the same ground-glass plate as the test field. The test stimulus consisted of a $1^{\circ}$ circular image, presented in the center segment of the adapting field, and the adapting field was also circular, subtending $3^{\circ}$ in visual angle. Thus, the subject could see the two stimulus fields concentrically through the observational window.

The test light pulse, whose maximum luminance was $990 \mathrm{~cd} / \mathrm{m}^{2}$, was presented with the 1 - and 300 -msec durations. Ten adapting luminance levels were tested: $0.04,0.13,0.40,1.26,3.50,12,36$, 115,316 , and $1,148 \mathrm{~cd} / \mathrm{m}^{2}$. These luminances were controlled by a series of calibrated Hoya neutral density glass filters. No special fixation device was used; therefore, the subject was instructed to fixate monocularly with his right eye at the center of the adapting field.
Procedure. A great part of the procedure was identical with that of Experiments 1 and 2. The subjects served in five experimental sessions, and one session was run per day. Within one session, 2 of the 10 adapting luminances were randomly selected for each subject, and then two RT vs. L curves, one with the 1 -msec duration and the other with the 300 -msec duration, were measured for each of the adapting luminances chosen. Before each experimental session, the subjects were given 20 warm-up RT trials.

After a period of about 10 min for dark adaptation, the darker adapting luminance was always first examined. At each adapting level, six to eight luminances for the test stimulus were determined at each stimulus duration by a criterion of $100 \%$ detection. Therefore, the test light pulses were always seen to be brighter than the adapting stimulus. The measurement procedure of RTs to these test luminances was the same as in Experiments 1 and 2. During the intersession interval, the subjects rested for about $2 \mathrm{~min}$. The second session began after about $5 \mathrm{~min}$ of preadaptation to the brighter adapting level. Other aspects of the procedure were the same as in Experiments 1 and 2.

Subjects. Three subjects, T.M., T.U., and Y.O., served in this experiment. New subjects, T.M. and Y.O., were trained to an asymptotic $\mathrm{RT}$ level in the present apparatus.

\section{Results}

RT vs. $L$ curve. Parts A-C of Figure 5 show an example of the data obtained for subject T.M: the geometric RT means are plotted against $\log \mathrm{L}$ at three adapting luminances and two durations of stimulus. The family of solid curves fitted to the data points represents the function used by Bartlett and MacLeod (1954):

$$
\mathrm{RT}=\frac{1}{\mathrm{k}\left(\log \mathrm{L} / \mathrm{L}_{0}\right)}+\mathrm{RT}_{0}
$$

where $\mathrm{k}$ is a constant and $\mathrm{L}_{0}$ represents a limiting luminance at which RT approaches infinity. The values of $\mathrm{RT}_{0}$ were graphically estimated from the asymptotes of RT vs. $\log \mathrm{L}$ curves. The values of $k$ and $L_{0}$ were then calculated by using the method of least squares.

The parameter $L_{0}$ in Equation 4 indicates whether the Weber generalization $\left(L_{0}\right.$ proportional to the luminance of the adapting field) applies over the luminance range explored. In Figure 5D, the estimated $\log$ values of $L_{0}$ for subject T.M. are plotted against log adapting luminance, and the straight lines represent a line of 1 slope. At the 1 -msec duration, the Weber-Fechner relationship holds up to $3.5 \mathrm{~cd} / \mathrm{m}^{2}$, whereas it continues up to $1.26 \mathrm{~cd} / \mathrm{m}^{2}$ at the $300-\mathrm{msec}$ duration. Part $\mathrm{E}$ shows the estimated $\mathrm{RT}_{0}$ value plotted against log adapting luminance for subject T.M., and it will be seen that the $\mathrm{RT}_{0}$ under the 300-msec duration condition has a slightly decreasing tendency as the adapting luminance increases. The results of subjects, T.U. and Y.O., were also analogous to T.M.'s data.

Critical duration. The luminance value required to produce a $\mathrm{RT}-\mathrm{RT}_{0}$ value of $50 \mathrm{msec}$ was computed from an individual fitted curve for each of the three subjects, and then averaged at each adapting level 

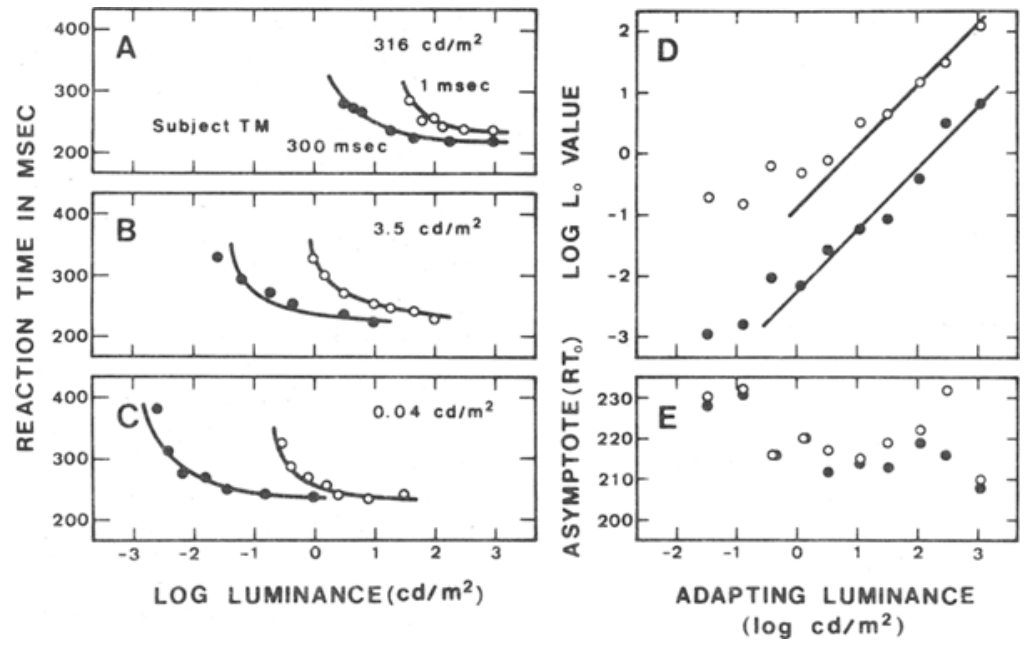

Figure 5. (A-C) Reaction time as a function of log luminance, with adapting luminance and pulse duration as a parameter for subject T.M. The solid curves running among the data points represent the RT vs. $\log L$ curve, Equation 4 , fitted by the method of least squares. Open circles are for 1-msec duration and filled circles for 300-msec duration. (D) Estimated parameter $L_{0}$ in Equation 4 as a function of adapting luminance for subject T.M. Open circles are for 1-msec duration and filled circles for 300-msec duration. The straight lines drawn through the data points represent a line of 1 slope. (E) Estimated asymptote $\left(R T_{0}\right)$ in Equation 4 as a function of adapting luminance for subject T.M. The legends are the same in part D.

and each stimulus duration. The upper part of Figure $6 \mathrm{~A}$ shows that the means of these calculated luminances are plotted as a function of log adapting luminance, taking stimulus duration as a parameter. At low adapting levels, the luminances are almost constant, but they increase linearly with the increased adapting luminance: there are ranges of luminances which approximate the prediction (the straight line of 1 slope) from the Weber-Fechner relationship. It can be found, however, that the ranges are curtailed by decreasing the stimulus duration.

The lower part of Figure 6B shows log critical duration plotted against log adapting luminance; the critical durations are based on the difference between the 1-msec duration data (open circles, Figure 6A) and the 300-msec duration data (filled circles, Figure 6A). It will be seen that the critical duration decreases as the adapting luminance increases.

\section{DISCUSSION}

\section{Parameters in RT vs. L Curve}

The data obtained in Experiments 1 and 2 indicate that the exponent $\mathrm{n}$ in Equation 3 changes with pulse duration, target size, and retinal position of the stimulus. The relationship of the exponent, pulse duration, and target size can be found in Part D of Figure 1: at the 300-msec duration, the values of $\mathrm{n}$ range from 0.35 to 0.40 over the range from 7.1

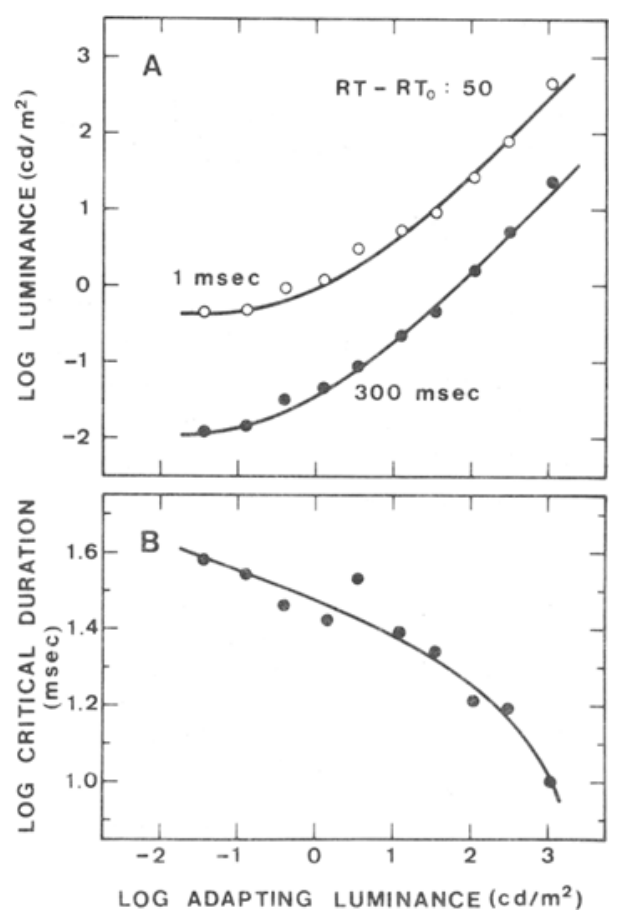

Figure 6. (A) Effect of adapting luminance on RT. Luminance required to produce a criterion $R T$ of $50 \mathrm{msec}$ above the asymptotic $\mathrm{RT}_{0}$ level is plotted as a function of adapting luminance for short duration (open circles) and long duration (filled circles). Each point represents the average of three subjects, T.M., T.U., and Y.O. The solid curves connecting the data points are drawn by visual inspection. (B) Critical duration as a function of adapting luminance. The solid curve is drawn by visual inspection. 
$1060^{\prime}$, which is consistent with the previous RT studies (Mansfield, 1973; Ueno, 1977; Vaughan et al., 1966); at the 1-msec duration, the $n$ values are always higher than those at the 300 -msec duration; and the exponents increase rapidly for targets less than 7.1' at both the durations.

For the $2.7^{\prime}$ target, the exponents estimated for the $1-\mathrm{msec}$ pulse were $1.17 \pm 0.13$ for subject A.H. and $1.33 \pm 0.21$ for subject T.U.; at the $300-\mathrm{msec}$ duration, the exponents were $0.58 \pm 0.08$ for A.H. and $0.43 \pm 0.06$ for T.U. The 300 -msec pulse data approximate Mansfield's (1973) finding that the exponent is about $1 / 2$ for a $0.05^{\circ}$ target whose area lies within Ricco's area of spatial summation. From Figure 1E, it is clear that the asymptotic $\mathrm{RT}_{0}$ values in Equation 3 roughly correspond to the changes in the exponent $\mathrm{n}$.

The RT vs. L curves in Experiment 2 are concerned with spatial information of red cones, since the 640-nm monochromatic light was used and retinal position was parametrically varied. It appears, therefore, that the determining factor in the RT is the density of cones at the particular locus stimulated. Figure 3D describes how the exponent $n$ in Equation 3 changes with distance from the fovea: the $n$ values are low at the foveal region and approximately $1 / 3$ at the $300-\mathrm{msec}$ duration; the exponents increase with increasing distance from the fovea; the exponents for the 1-msec pulse are always higher than those for the 300 -msec pulse, which resembles the finding in Experiment 1. Furthermore, it is clear from Figure $3 \mathrm{E}$ that the effect of retinal locus on the asymptotic $\mathrm{RT}_{0}$ is more noticeable. The primary determinant of $\mathrm{RT}_{0}$ seems to be the total number of the cones involved at different retinal positions, because Figure $3 \mathrm{E}$ indicates that the spatial distribution of the estimated $\mathrm{RT}_{\mathrm{D}}$ values inversely corresponds to the $\emptyset$ sterberg count of cone density in the retina (Rodieck, 1973, p. 351).

The parameter $L_{0}$ in Equation 4 is of special significance in examining the effect of adapting luminance on the RT. Part D of Figure 5 offers evidence that the $L_{0}$ represents the Weber fraction: at adapting levels lower than $1.26 \mathrm{~cd} / \mathrm{m}^{2}$, especially at a shor duration $(1 \mathrm{msec})$, there will always be an adapting luminance sufficiently dim to exert no effect on the $\mathrm{RT}$; at higher adapting luminances, the $\log \mathrm{L}_{0}$ value lies on a straight line of 1 slope in $\log$ - $\log$ coordinates, satisfying the Weber-Fechner relationship. This finding agrees closely with the results of threshold experiments (e.g., Barlow, 1957; Boynton \& Whitten, 1970).

Figure $5 E$ shows that the asymptotic $R T_{0}$ for the 300 -msec pulse tends slightly to the decrease with the increased adapting luminance. This result is inconsistent with that of Bartlett and MacLeod (1954), who found that the $\mathrm{RT}_{0}$ values in Equation 4 show a nearly linear increase with increasing log adapting luminance. The difference between the present result and Bartlett and MacLeod's may be due 10 the luminance range of the test stimulus. Bartlett and Macleod always used a constant luminance $(3.18 \log \mathrm{mL})$ as the highest luminance of the test target at three different adapting levels, whereas the highest luminance employed in the present case was varied with the decreasing adapting luminance, as is clear from Parts A-C of Figure 5.

\section{Effect of Target Size}

Figure 2A shows the effect of target size on the criterion luminance that was required to produce a RT of 50 msec greater than the asymptotic level. For target sizes less than $10^{\circ}$, the calculated criterion luminance obeys Ricco's law, and for larger targets it remains constant, independent of target size. This upper limit of spatial summation is not always large, compared with the data of previous threshold studies on cone summation (Hallett, 1963). Another interesting finding shown in Figure $2 \mathrm{~A}$ is that the stimulus pulse durations do not exert any effect on the critical angle of $10^{\prime}$. However, further examinations would be needed with respect to this point, because prior threshold data have indicated that the upper limit of complete spatial summation is decreased by increasing the duration of the stimulus (e.g., Barlow, 1958).

Figure $2 \mathrm{~B}$ shows the critical duration as a function of stimulus area. The most significant finding is that for areas less than 13.4', the critical duration increases with decreasing area, though the effect is small. This result agrees with the threshold data obtained at the fovea by Karn (1936), implying a sensitive capacity of the RT measure for spatial integration.

\section{Effect of Retinal Locus}

Figure $4 \mathrm{~A}$ shows the effect of retinal locus on the criterion luminance at the same $\mathrm{RT}-\mathrm{RT}_{0}$ level as in Experiment 1. At the fovea or parafovea, the calculated luminance becomes lowest and increases with increasing distance from the fovea. Taking reciprocals of the luminance values, it is found that the shape of the curves in Figure 4A is similar to the sensitivity curve of the photopic RT component in the data of Rains (1963) as analyzed by Mansfield (1973). However, there is a difference between the present curve and Rains-Mansfield's curve: the luminance in the nasal hemiretina is high (low sensitivity). as shown in Figure $4 \mathrm{~A}$, relative to that in the temporal hemiretina; on the other hand, RainsMansfield's curve is approximately symmetric about the fovea. The reason for this discrepancy is not clear.

Part B of Figure 4 shows the critical duration as a 
function of eccentricity. At the fovea, the critical duration is shortest and increases pointedly with distance from the fovea. It is conceivable that the 640-nm monochromatic light employed, together with the spatial distribution of cone density on the retina, reflects the characteristics revealed in Figure 4B. The total number of cones stimulated by the $1^{\circ}$ target stimulus is expected to be more at the fovea than at the periphery. To produce a constant visual effect, therefore, it would be assumed that the electrical signals from the fovea are summated more rapidly in comparison with those from the periphery, and, consequently, the critical duration becomes shorter at the fovea.

\section{Effect of Adapting Luminance}

Figure 6A presents the effect of adapting luminance on the luminance required to produce a RT difference, $\mathrm{RT}-\mathrm{RT}_{0}$, of $50 \mathrm{msec}$. As expected, the curves drawn through the data points correspond closely to the shape of the $\mathrm{L}_{0}$ values shown in Figure 5D. Furthermore, the shape of the curves in Figure $6 \mathrm{~A}$ is very similar to the results of threshold (Barlow, 1957) and electrophysiological studies (Boynton \& Whitten, 1970). This fact indicates that the RT measure, together with Bartlett and MacLeod's equation, becomes a powerful tool for investigating brightness discrimination.

In Figure 6B, the calculated critical duration is plotted as a function of adapting luminance. The curve connecting the data points can broadly be compared with the shape of the curves obtained from the threshold experiments which examined the relation between threshold increment and adapting luminance (e.g., Barlow, 1958; Biersdorf, 1955; Graham \& Kemp, 1938; Keller, 1941; Roufs, 1972).

Finally, it is necessary to make a comment about a methodological aspect of the present investigation. If $\mathrm{RT}$ - $\mathrm{RT}_{0}$ values other than $50 \mathrm{msec}$ were taken as criterion responses to examine temporal summation at suprathreshold levels, then the resulting critical durations would be different from those in the present case. In fact, recent RT studies have demonstrated that the critical duration is not uniquely determined, but rather is a function of the RT - RT level (Ueno, 1976a, 1977). Nevertheless, preliminary attempts found that the direction of effects of the three variables, i.e., target size, retinal locus, and adapting luminance, on the critical duration was invariant under the change in $R T-R T_{0}$ level.

\section{REFERENCES}

BARLOW, H. B. Increment thresholds at low intensities considered as signal/noise discriminations. Joumal of Physiology, 1957, 136, 469.488.

BARLOW, H. B. Temporal and spatial summation in human vision at different background intensities. Journal of Physiology, 1958. 141. 337-350.

Bartlett, N. R., \& MACLEod, S. Effect of flash and field luminance upon human reaction time. Journal of the Optical Society of America. 1954, 44, 306-311.

Baumgardt, E., \& Hillmann, B. Duration and size as determinants of peripheral retinal response. Journal of the Optical Society of America, 1961, 51, 340-344.

Biersdorf, W. R. Critical duration in visual brightness discrimination for retinal areas of various sizes. Journal of the Optical Society of America, 1955, 45, 920-925.

Boynton, R. M. \& WhITEN, D. N. Visual adaptation in monkey cones: Recordings of late receptor potentials. Science, 1970, 170. 1423-1426.

Graham, C. H., \& KeMP, E. H. Brightness discrimination as a function of the duration of the increment in intensity. Journal of General Physiology, 1938, 21, 635-650.

Grossberg, M. Frequencies and latencies in detecting two-flash stimuli. Perception \& Psychophysics, 1970, 7, 377.380.

Hallett, P. E. Spatial summation. Vision Research, 1963, 3, 9-24.

HaRTLINE, H. K. Intensity and duration in the excitation of single photoreceptor units. Journal of Cellular and Comparative Physiology, 1934, 5, 229-247.

HERRICK, R. M. Foveal luminance discrimination as a function of the duration of the decrement or increment in luminance. Journal of Comparative and Physiological Psychology, 1956, 49. 437-443.

KARN, H. K. Area and intensity-time relation in the fovea. Journal of General Psychology, 1936, 14, 360-369.

KELLER, M. The relation between the critical duration and intensity in brightness discrimination. Journal of Experimental Psychology, 1941. 28, 407-418.

Kietzman, M. L., \& Gillam, B. J. Visual temporal integration and simple reaction time. Perception \& Psychophysics, 1972, 11. 333-340.

Krauskopf, J., \& Mollon, J. D. The independence of the temporal integration properties of individual chromatic mechanisms in the human eye. Journal of Physiology, 1971, 219, 611-623.

LIPETZ, L. E. The transfer functions of sensory intensity in the nervous system. Vision Research, 1969. 9, 1205-1234.

Mansfield, R. J. W. Latency functions in human vision. Vision Research. 1973, 13, 2219-2234.

PrÉRon, H. The sensations. (Trans. M. H. Pirenne \& B. C. Abbot) New Haven: Yale University Press, 1952.

RaINs, J. D. Signal luminance and position effects in human reaction time. Vision Research, 1963, 3, 239-251.

Rodieck, R. W. The vertebrate retina. San Francisco: Freeman, 1973.

Rours, J. A. J. Dynamic properties of vision-I. Experimental relationships between flicker and flash thresholds. Vision $R e^{-}$ search, 1972, 12, 261-278.

UENO, T. Luminance-duration relation in reaction time to spectral stimuli. Vision Research, 1976, 16, 721-725. (a)

UENo, T. Temporal summation and reaction time to double-light pulses at suprathreshold levels. Perception \& Psychophysics. 1976, 19, 399-404. (b)

UENO, T. Reaction time as a measure of temporal summation at suprathreshold levels. Vision Research, 1977, 17, 227-232.

UetsukI, T.. \& IKEDA, M. Adaptation and critical duration for Stiles $\pi$ mechanisms. Journal of the Optical Society of America, $1971,61,821-828$.

Vaughan, H. G., Jr., Costa, L. D., \& Gilden, L. The functional relation of visual evoked response and reaction time to stimulus intensity. Vision Research, 1966, 6. 645-656.

(Received for publication May 6, 1977; revision accepted August, 25, 1977.) 